of high status, and in many cases the use of iron in combination with other metals. A rather different picture is presented in India, where Chakrabarti (Antiquity 50, $114 ; 1976)$ has argued for an independent growth of iron-working by $800 \mathrm{BC}$. Here, however, the range of iron goods is predominantly domestic, and a phase of gradual development has yet to be documented. It is possible that the earliest stages of iron-working in the subcontinent have yet to be explored as the date of 1025 $\pm 110 \mathrm{BC}$ reported by Jain (Prehistory and Protohistory of India, 191; 1979) for a layer containing small iron tools would support the suggestion of a more extended development. Even more problematical is the question of the origin of iron in west Africa. The technology described by Tylecote (W. Afr. J. Archaeol. 5, 1; 1975) from the Nok culture of Nigeria is already well developed by about the fourth century $\mathrm{BC}$, too early to be derived from any of the known industries of the north-east in Egypt or the Sudan. The solution proposed is that the technology diffused from the north coast across the Sahara but the possibility of independent invention cannot be ruled out. There was no previous tradition of metallurgy but the ready availability of high-grade ores and the pyrotechnic skill demonstrated by the Nok clay figurines fulfil some of the necessary preconditions.

The theory of a unique invention of iron technology is clearly now very difficult, if not impossible, to maintain, and the focus of research has moved from questions of diffusion to the conditions under which the technology developed. Although the west African case still remains problematic, the other areas all shared a competent bronze metallurgy and in most of them the use of early iron is strongly associated with high status objects. The development of iron technology may therefore be seen as taking place in stratified societies where considerable technological resources are invested in producing prestige items. This stage of early development is quite distinct from a later phase when iron replaces bronze for a wide range of everyday items. Snodgrass (Dark Age of Greece, $213 ; 1971$ ) argued, on the basis of limited analyses of early iron objects, that they were in fact not superior to the quality of bronzes but actually inferior and he attributed the wide-scale adoption of iron in Greece to an interruption in the supply of raw materials forcing the use of local resources. Maddin, Muhly and Wheeler (Scient. Amer. 237, $122 ; 1977)$ have described the discovery of the essential techniques of carburization, quenching and tempering in the Near East, but they too, like Waldbaum, ascribe the demise of bronze to a breakdown in the supply of raw materials, especially tin. Further analyses are needed of both iron and bronze objects, but it is now recognized that the problems of the discovery and adoption of iron can only be understood in relation to contemporary social and economic conditions.

\title{
Ocean trench topography
}

\section{from D.L. Turcotte}

THE anomalous topography and negative gravity anomalies of ocean trenches are readily explained by plate tectonics. The oceanic lithosphere bends and descends into the interior of the Earth in a process referred to as subduction. This sounds relatively straightforward until one realises that the oceanic lithosphere is a $100 \mathrm{~km}$ thick plate made up of cool, brittle rock. How can a plate $100 \mathrm{~km}$ thick made up of a brittle material be bent through an angle of $45^{\circ}$ or more in the subduction process?

Two recent papers examine alternative hypotheses for the bending behaviour of the lithosphere. Chapple and Forsyth $(J$. geophys. Res. 84, 6729; 1979) argue in favour of an elastic-perfectly plastic rheology. In the upper $20 \mathrm{~km}$ they assume that the rock is relatively weak due to low lithostatic pressure (the weight of the overlying rock) and postulate a yield stress of 1 kbar. In this region the tensional bending stresses due to the flexure of the lithosphere at an ocean trench give the normal faulting earthquakes observed seaward of many ocean trenches. Between depths of 20 and $50 \mathrm{~km}$ a yield stress of 6 $\mathrm{kbar}$ is postulated. At stresses less than 6 kbar the rock is assumed to behave elastically and then yields freely when the yield stress is reached. This is the definition of an elastic-perfectly plastic rheology. It is this part of the lithosphere which transmits the high stresses associated with the bending. At depths greater than $50 \mathrm{~km}$ temperatures are assumed to be sufficiently high that thermally-activated creep processes (that is, dislocation creep) relieve the elastic stresses. Predicted trench topography agrees well with observations.

The primary objection to the application of the elastic-perfectly plastic rheology is the high stress level required. Many seismologists object to high stresses (greater than a few kilobars) in the lithosphere. Seismic studies indicate that the stress drops in most large earthquakes are of the order of 100 bar or less. Stress fields derived from the surface strain associated with earthquakes give similar values. On the other hand, scientists who study the mechanical behaviour of rock in the laboratory tend to favour high stresses. Mantle rocks tested in the laboratory can easily be stressed to $5 \mathrm{kbar}$ without failure. Extrapolations of laboratory derived friction laws and creep studies also give high stresses in the lithosphere.

An alternative approach to the problem of the bending of the oceanic lithosphere at ocean trenches has recently been given by Melosh and Raetsky (Geophys. J. 60, 333; 1980). These authors postulate that the oceanic lithosphere is made up of a near-

D.L. Turcotte is Professor of Geological Sciences, Cornell University, Ithaca, New York, and is currently on leave at the Department of Geology and Mineralogy, Oxford. surface elastic layer with a thickness of $15-30 \mathrm{~km}$ overlying a highly viscous layer $50-80 \mathrm{~km}$ thick. The shape of the bending lithosphere is primarily attributed to the viscous flow in the lower lithosphere. The viscous flow is attributed to solid-state creep. Again good agreement with the observations is obtained. The maximum stress required is a few hundred bars.

The principal advantage of the viscous rheology is the lower required stress level. However, the derived viscosities of $2 \times 10^{22}-10^{23}$ poise are quite low, being less than an order of magnitude larger than the viscosity associated with solid-state creep in the deeper mantle. This does not seem to be consistent with the temperature dependence of thermally activated creep processes.

It is clear that comparisons with observations at oceanic trenches cannot differentiate between the elastic-perfectly plastic and elastic-viscous hypotheses. The alternative rheologies must be applied to other flexure problems. Examples include the flexure of the lithosphere under the load of oceanic islands, the load of sediments, and the load of mountain belts. It is implicit in the viscous analysis that bending stresses will relax with time. Whether the resultant deformation of the lithosphere when subjected to a fixed load can be observed is also a subject of controversy.

An understanding of the bending behaviour of the lithosphere under load will help in our understanding of how stress is transmitted through it. An essential part of this understanding is the stress level present. At present the stress level at depth in the lithosphere is uncertain by more than an order of magnitude. Only when we understand the state of stress in the lithosphere will we be able to start to understand in some detail why earthquakes occur.

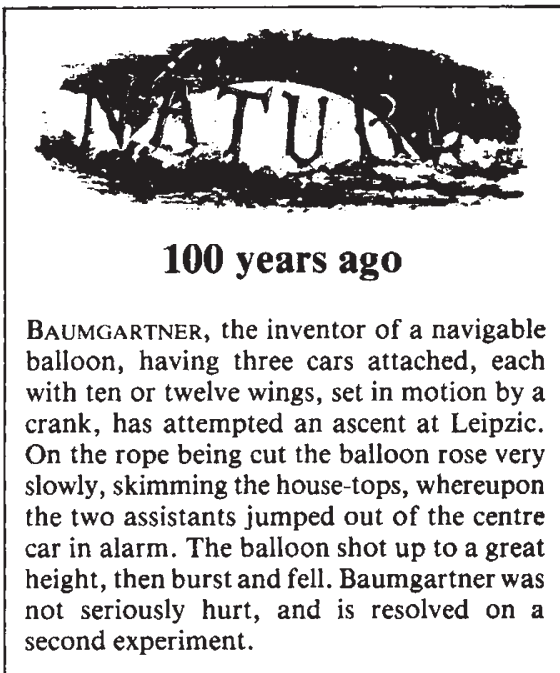

From Nature 21, 8 April, 549; 1880. 